\title{
Indirect control of DSRs for regulating power provision and solving local congestions
}

Sossan, Fabrizio; Marinelli, Mattia; Costanzo, Giuseppe Tommaso; Bindner, Henrik W.

Published in:

Proceedings of the 4th International Youth Conference on Energy 2013

Publication date:

2013

Link back to DTU Orbit

Citation (APA):

Sossan, F., Marinelli, M., Costanzo, G. T., \& Bindner, H. W. (2013). Indirect control of DSRs for regulating power provision and solving local congestions. In Proceedings of the 4th International Youth Conference on Energy 2013

\section{General rights}

Copyright and moral rights for the publications made accessible in the public portal are retained by the authors and/or other copyright owners and it is a condition of accessing publications that users recognise and abide by the legal requirements associated with these rights.

- Users may download and print one copy of any publication from the public portal for the purpose of private study or research.

- You may not further distribute the material or use it for any profit-making activity or commercial gain

- You may freely distribute the URL identifying the publication in the public portal

If you believe that this document breaches copyright please contact us providing details, and we will remove access to the work immediately and investigate your claim. 


\title{
Indirect control of DSRs for regulating power provision and solving local congestions
}

\author{
Fabrizio Sossan, Mattia Marinelli, Giuseppe Tommaso Costanzo and Henrik Bindner \\ Center for Electric Power and Energy \\ Technical University of Denmark \\ 4000 Roskilde, Denmark \\ \{faso, matm, guco, hwbi\}@elektro.dtu.dk
}

\begin{abstract}
Inducing a shift in the electricity consumption using a broadcasted dynamic price for the energy is often proposed as a resource for providing regulating power and it is becoming an increasing research focus for enabling higher penetration of renewable energy in the current power system.

This paper shows how using indirect control (or control by price) without any precautions, might easily lead to congestions in nearly saturated distribution grids.

An auto tuning local controller which acts on the price signal at distribution level is proposed for solving the congestion. Simulations are performed with the CIGRE' MV reference network with 346 electrically heated buildings as Demand Side Resources, DSRs. The dynamic hourly price of the regulating power provided by Nord Pool Spot market has been used as indirect control signal for the flexible demand.
\end{abstract}

Keywords - Power distribution, Power demand, Smart grids, control-by-price, congestions

\section{INTRODUCTION}

Exploiting the flexibility at demand side level in the power system is becoming of increasing focus in order to allow higher penetration of variable renewable energy and relocate ancillary services. Electric loads which are suitable to be controlled for providing regulating power are the ones whose power consumption can be deferred without compromising the primary services they are supplying to the users. Such units are commonly called Demand Side Resources, DSRs.

Indirect control, or control by price, consists of inducing a shift in the power consumption of DSRs using a broadcasted control signal and it is often proposed for providing regulating power to the power system [1]. The control signal sent to the DSRs is usually the price for the electric energy because it gives to the users a clear and identifiable economic advantage for moving its power consumption [2]. Indirect control allows to simplify the complex task of controlling a big population of units and it requires simple ICT infrastructure[3] since it is realized using one way communication with the units. On the other hand, the response given by indirect control is not known at priori because the reaction of the units to the indirect control signal is on spontaneous basis and according local preferences and so the actual support to the power system can only be estimated with uncertainty [4], [5].

In this paper it is shown how using indirect control for supplying regulating power from DSRs in a nearly congested distribution network can easily lead to congestions especially when low prices are aligned with peak of conventional demand. This happens because indirect control induces the DSRs to a coherent and coordinated use of electric power. As solution for this inconvenient, a local auto tuning controller that acts on the price signal at distribution level is proposed. Simulations that show the efficacy of the proposed solution are reported in the paper.

A similar work have been proposed in [6] on a smaller two nodes grid and with a regulator where the gains are statically assigned. The here proposed auto tuning feature of the substation regulator is interesting because it allows the controller to be replicated and placed anywhere in the network without need of redefining the gains. Furthermore auto tuning capabilities have to be addressed in case of deployment of such solutions.

CIGRE' medium voltage reference network is used in the proposed simulations. The population of controllable DSRs are buildings heated by electric space heating. The indirect control signal is the one hour price for the regulating power taken from the Nord Pool Spot market. The penetration of nominal flexible demand at substation level is $43 \%$ (calculated as ratio between flexible demand and flexible demand plus conventional consumption).

Power system simulations are performed in Matlab Simulink and the DSRs population is simulated using Python. The simulation setup together with the proposed congestion controller are discussed in Section II. Section III presents the results. Discussion of results is provided in Section IV. Finally, conclusions are provided in Section IV.

\section{Methods}

This section describes the simulation setup and the substation auto tuning controller for congestions management.

Simulation scenario is composed by a population of 346 Demand Side Resources whose power consumption is indirect controlled with the hourly regulating power price from Nord Pool Spot market. The demand side units are simulated using a Python based simulation platform and they are spread at the distribution level in the medium voltage CIGRE' reference network. Conventional residential and commercial loads with fixed daily consumption patterns are also present in the distribution network.

Network simulations have been performed in Matlab Simulink using SimPowerSystem libraries. A link between Python and Matlab has been established in order to allow data exchange. 


\section{A. DSRs population}

The controllable demand side population is composed by 346 buildings equipped with electric radiators for space heating. Each building has a nominal heating power capability of $10 \mathrm{~kW}$ and it is simulated individually using a second order model for describing thermal dynamics. In order to represent a heterogeneous population, the parameters of the models have been slightly variated around nominal values following a normal distribution for accounting variation in size and insulation characteristics.

The nominal thermal model has been built using grey box modeling on a real building [7], Power Flexhouse [8].

Power Flexhouse is an automated 10 rooms, electrically heated, office building which belongs to SYSLAB, the distributed power system facility of DTU Ris $\emptyset$ campus.

power Flexhouse thermal model is described by a system of differential equation reported in Equation 1 and Equation 2. The two temperature states are for the indoor air, $T^{i n}$, and heaters surfaces. The electric power $P$ is the controllable input. The disturbances are the solar radiation $S$ and the outdoor temperature $T^{o}$. For simulations, the model is discretized with a sampling time of $300 \mathrm{~s}$.

$$
\begin{aligned}
\underline{\dot{x}} & =\left(\begin{array}{cc}
-2.73 \times 10^{-5} & 9.15 \times 10^{-6} \\
1.48 \times 10^{-3} & -1.48 \times 10^{-3}
\end{array}\right) \underline{x} \\
& +\left(\begin{array}{ccc}
0 & 1.81 \times 10^{-5} & 7.38 \times 10^{-4} \\
1.48 \times 10^{-2} & 0 & 0
\end{array}\right)\left(\begin{array}{c}
P \\
T^{\text {out }} \\
S
\end{array}\right) \\
T^{i n} & =\left(\begin{array}{ll}
1 & 0
\end{array}\right) \underline{x}
\end{aligned}
$$

Each building is controlled with a simple control algorithm that, indirectly, adjusts the electric energy consumption according to the received price signal: the controller, whose formula is shown in Equation 3, produces an offset of the indoor temperature set point (Equation 4) at each instant of time $i$ when a new price, $p_{i}$, is available.

In Equation 4, $\bar{p}$ is the mean of the prices over the last 12 hours and $k$ is a coefficient for setting the responsiveness of the algorithm (it equals 10 in the proposed simulations).

The indoor temperature set point, Equation 4, is maintained using a simple thermostatic controller.

$$
\begin{aligned}
& \hat{p}_{i}=\frac{p_{i}-\bar{p}_{i}}{\bar{p}_{i}} \\
& T_{\text {set point }}^{\text {in }}=21^{\circ} \mathrm{C}-k \hat{p}_{i}
\end{aligned}
$$

Roughly, Equation 3 and Equation 4 tell that if the current price is higher than the average of the past ones, the indoor set point is decreased and vice-versa. It is worth noting that such algorithm has a null contribution at steady state, so no deviation to the indoor reference is applied when the price is constant for sufficient long time (12 hours in this case).

\section{B. Electric network layout}

The network used for the study is the CIGRE' MV European Reference. A comprehensive description of this network can be found in reference [9]. For sake of clarity, the main aspects are here reported. The European MV distribution feeders are three-phase and either of meshed or radial structure, with the latter dominating rural installations. The benchmark allows flexibility to model both meshed and radial structures.
Each feeder includes numerous laterals at which MV/LV transformers could be connected. The nominal voltage is 20 $\mathrm{kV}$ and the system frequency is $50 \mathrm{~Hz}$. The study has been performed in the feeder 1 (urban), whose main information are here listed:

- $\quad 11 \mathrm{MV}$ buses and $11 \mathrm{LV}$ (Low Voltage) buses;

- $4590 \mathrm{kVA}$ of transformer MV/LV capacity;

- $\quad 2.82 \mathrm{~km}$ OHL (OverHead lines) and $12.2 \mathrm{~km}$ Cable lines.

Three configuration switches are present but for this study are left in open status.

At the current stage, $7 \mathrm{MV} / \mathrm{LV}$ substations out of 11 possible are used for simulations. Low voltage network in the substations is not represented in details. Substations are composed 3 controllable loads:

- lumped flexible demand load;

- lumped residential base load;

- lumped commercial base load (not present in all substations);

The consumption of the lumped flexible load corresponds to the aggregated consumption of a group of DSRs. The size of each group has been chosen according to the nominal power of the substation. The residential and commercial base loads are subject to predefined daily consumption patterns which are described in [9]; their (active) power consumption profile are shown in Figure 1 where $t=0 h$ refers to midnight. Because the flexible demand is assumed to be electric domestic space heating, the curve consumption of the conventional residential load has been scaled to $0.7 \mathrm{pu}$.

The global amount of electric consumption for each substation has been chosen, as shown in Section IV, in order to nearly saturate substations capacities during evening peak hours.

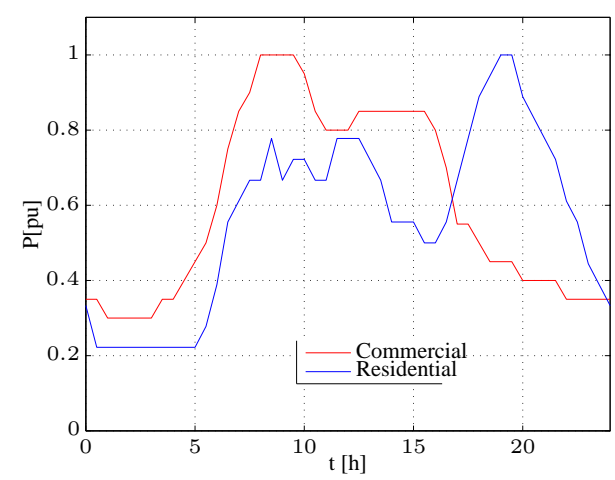

Fig. 1. Static daily patterns for the conventional residential and commercial consumption. Because flexible consumption is also domestic, residential consumption profile has been scaled in amplitude by a factor 0.7 .

\section{Congestion controller}

The overview of the implemented control approach is shown in Figure 2: a substation controller detects congestions (which occur when the power transit exceeds the substation transformer nominal value) and, in order to solve them, it produces an offset to add to the current price. Price price $_{i}$ 
is the price control signal which, as said in Section II, is the regulating power price from Nord Pool Spot market. Quantity $p_{i}$ is the energy price delivered to the electric loads underneath that substation. $P_{e_{i}}$ is the electric power transit in the substation transformer and it is composed both by conventional and flexible consumption. Subscripts $i$ refer to time instants. Electric power is here expressed in pu, per unit, and referred to the nominal power of the substation $\left(P[p u]=P / P_{n o m}\right)$ transformer according to the specification of the reference network.

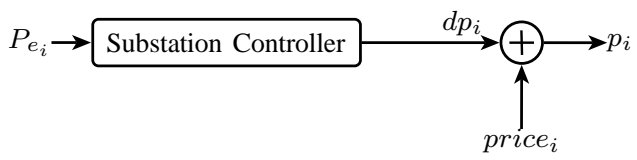

Fig. 2. General overview of the proposed control approach. The substation controller measures the power transit and it produces an additive offset $d p_{i}$ for the price price $_{i}$. Price $p_{i}$ is the one sent to the DSRs below this substation.

Considering Figure 2, if the sensitivity of the aggregated flexible demand ( $s_{i}$ and defined in Equation 5) to an unitary variation of the price signal is known at each instant of time $t=i$, then the amount of overload could be measured and multiplied by $s$ in order to get the exact variation in price needed to restore the power transit below its nominal value acting on flexible loads. The problem is that the quantity sis function of time and states of the DSRs for a given substation and therefore, considered the characteristics of indirect control (one way communication), it is unknown.

$$
\begin{aligned}
& s_{i}=\frac{\Delta \text { price }_{i}}{\Delta P_{e_{i}}} \\
& \Delta P_{e_{i}}=P_{e_{i}}-P_{e_{i-1}} \\
& \Delta \text { price }_{i}=\text { price }_{i}-\text { price }_{i-1}
\end{aligned}
$$

The form of the proposed controller is a closed loop PI regulator whose input is the deviation from the nominal power, $1 \mathrm{pu}$; its diagram is shown in Figure 3.

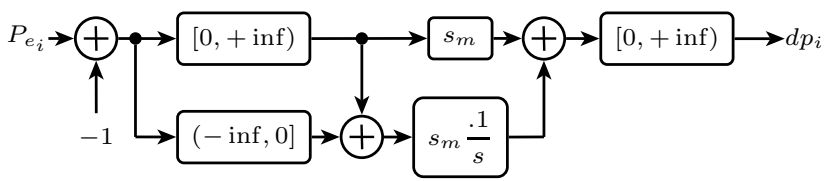

Fig. 3. The proposed controller that produces an overprice $d p$ for the loads in each substation when a congestion $\left(P_{e}>1\right)$ occurs. The gain $s_{m}$ is self tuned with the strategy described in Section II.

The gain of both proportional and integral parts is proportional to $s_{m}$. Coefficient $s_{m}$ is defined in Equation 6 as the smallest variation in the observed (past) aggregated power consumption for a unit of price.

$$
s_{m}=\min \left\{s_{i}\right\}
$$

The aim of the proportional part of the regulator is to give a one shot guess (given by $s_{m}$ times the amount of overload) of the deviation in price to send to the units. In general, the guess will not be able to solve the congestion because, as said before, the exact value $s_{i}$ would be needed. In particular here, $s_{m}$ is chosen as the smallest value among a set of past $s_{i}$ and it might easily overestimate indirect control capability leading to an offset price which is not high enough. The task of the integral part of the regulator is indeed to correct the proportional action and produce a higher price in order to try to restore the nominal power transit.

The input of the proportional part of the regulator is limited to the interval $[0,+$ inf) because it has to act only when a congestion is occurring. However a negative input (- inf, 0$]$ is allowed for the integral part of the controller in order to remove the offset price when no congestion is occurring.

The offset price produced by the controller is in the interval $[0,1)$, i.e. the controller can only raise the price and deincetive consumption. The integral part of the controller has an anti wind-up loop which is not shown in the diagram of Figure 3.

It is worth noting that the parameter $s_{i}$ in Equation 5 is not influenced by the conventional load which does not react to the price signal.

Congestion controllers are placed in all the seven substations and they adjust their gains automatically on line using Equation 6 with measurements of the power transit and the price signal for the regulating price. No manual individual tuning is needed for the controllers.

In order to allow the value $s_{m}$ to be updated with newer ones, the stored value of $s_{m}$ is multiplied by a forgetting factor equal to $e^{\lambda / 12}$ with $\lambda$ as the age of the parameters in hours.

In the proposed simulations, the substation controller can adjust the price signal once every 5 minutes.

\section{RESULTS}

Three simulation scenarios $a, b$ and $c$ have been selected. All present the same configurations for both the electric network (layout, power profiles, flexible demand disposition) and DSRs population (size, model parameters and algorithm parameters). The only difference in the scenarios is the price signal used for indirect control of the DSRs.

Here presented simulations last for 24 hours, starting from midnight of 2nd November 2012. All the time series used as input for the simulations are right aligned in time, meaning that both weather data (outside temperature and solar radiation), daily consumption patterns and price signal refer to the same time period. Coherently with the notation used before, the power transits in the plots are expressed in pu, that is transit power divided the nominal capacity of the substation transformer.

In scenario $a$, the price signal is kept constant. Because the demand side algorithm does not give any contribution at steady state, the power consumption of each DSR is only function of its state and it does not depend on the the absolute value of the price.

Scenario $a$ represents nowadays situation where flexibility present at demand side level is not exploited.

The power transit of each substation, from number 1 to 7 , and the price signal of scenario $a$ are shown in Figure 4.

In scenario $b$, the regulating power price from Nord Pool Spot market is used as indirect control signal for the flexible 
demand. Substations power transits and price signal are shown in Figure 5

In simulation scenario $c$, the congestion controller proposed in Section III is operating in the 7 substations. Results of scenario $c$ are shown in Figure 6 which shows the power transits and the price signals, this time one for each substation.

Substation 0 is not shown in the figures because the power transit never exceed the nominal value.
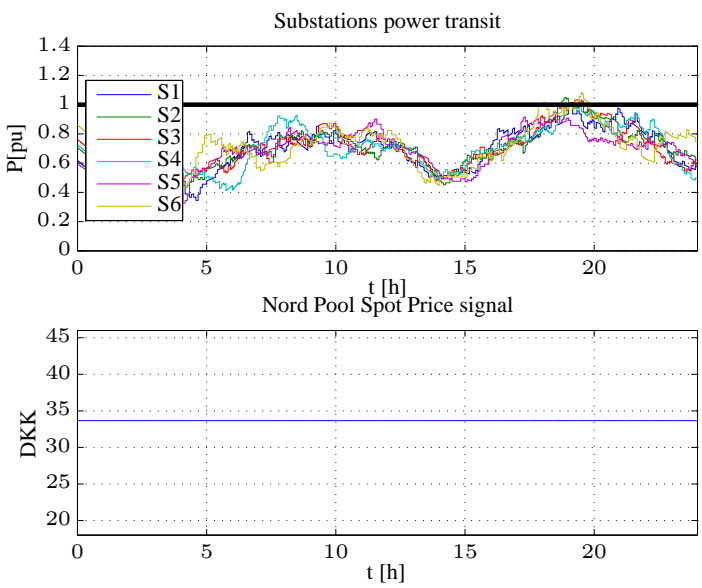

Fig. 4. Scenario $a$. The price signal is flat, or equivalently, there is no flexible consumption (nowadays situation). Substations are saturated (or nearly) during peak hours.
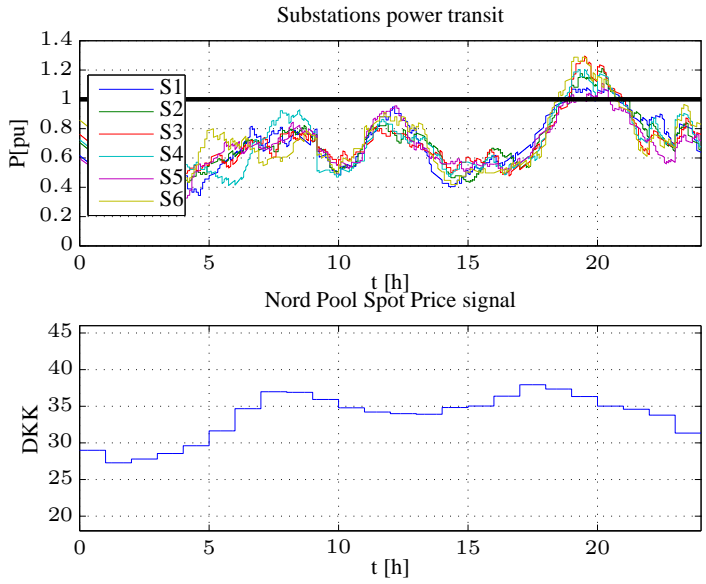

Fig. 5. Scenario $b$. All the substations are overloaded when the flexible demand is indirect controlled with the given Nord Pool Spot price signal for regulating power.

\section{Discussion}

\section{A. Results discussion}

Figure 4, 5 and 6 show the power transit at each substation respectively when: scenario $a$ ) the demand side units are not controlled at all, scenario $b$ ) they are controlled with a price signal, scenario $c$ ) they are controlled and the proposed substation regulator is active.

Scenario $a$ depicts a situation where all the substations are at the limit of their nominal capacity during evening hours,
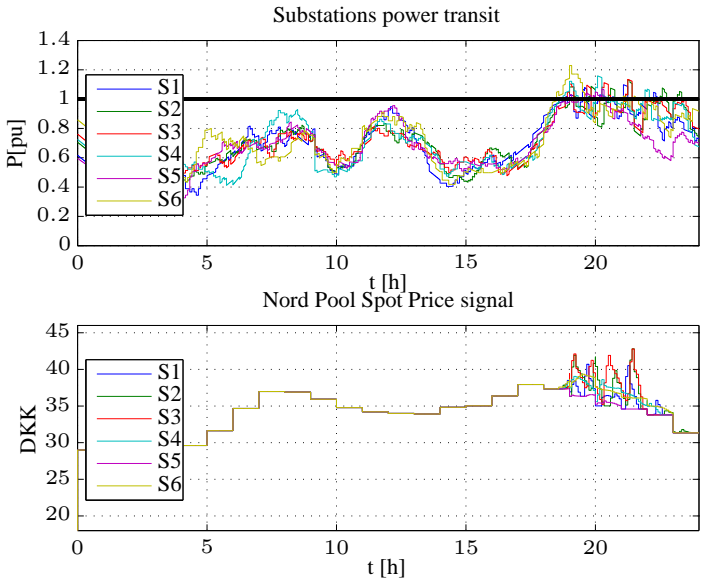

Fig. 6. Scenario $c$. The congestion controller is now implemented in all substations and for each of them is modifying Nord Pool Spot price in order to limit the congestions.

around $t=20 h$, when the peak of the domestic consumption is reached.

In scenario $b$, Figure 5, the dynamic price signal induces the flexible loads to shift their power consumption when the energy price is low. The coordinated electric power demand of the DSRs, in confluence with the not flexible consumption, increases the congestion level in all the substations.

Figure 5 shows how in scenario $c$, the substations controllers starts to raise the price signal as soon as they detect an overload; consequently the DSRs tend to reduce their power consumption. When no congestion is occurring, the controllers do not apply any price offset.

A closer inspection of Figure 6, during the time frame where the congestions happen, shows that 1 ) the power consumption oscillates around $1 \mathrm{pu}$ or 2) it slowly decreases to one, 3) the peak values are reduced but the 'wave' of high consumption last longer than scenario $b$.

- Case 1) occurs when the identified value $s_{m}$ for a certain substation is underestimating indirect control capability: in this case only the proportional regulator is raising the price. After the price is sent, the power transit is reduced below its nominal value: because the proportional regulator is not feeded anymore, the power consumption returns to a value higher than $1 \mathrm{pu}$ and the cycle starts again.

In case 2), the value $s_{m}$ is overestimating indirect control capability and the one shot guess done with the proportional action cannot remove the congestion: in this case the integral regulator starts to act and it increases gradually the price causing small decreasing steps in the power consumption.

Explanation for case 3 ) is that the controller, for solving the congestion, raises the price: once the congestion is solved, the controller removes the price offset causing the DSR to increase a little the consumption again; this sort of regulator wake effect concurs in keeping an high level of consumption even after the congestion is solved.

Table I reports some relevant information of the power transits of each substation. Data are referred to the time frame $\sim 18.5 h<t<\sim 20.7 h$ where congestion is occurring. The controller is able to trim the consumption peak values in all 
TABLE I. SUBSTATIONS POWER TRANSIT VALUES DURING CONGESTION

\begin{tabular}{cccccccc} 
Power transit & Scenario & S1 & S2 & S3 & S4 & S5 & S6 \\
\hline \hline \multirow{2}{*}{ peak value } & $b$ & 1.08 & 1.21 & 1.30 & 1.20 & 1.11 & 1.29 \\
& $c$ & 1.08 & 1.09 & 1.11 & 1.16 & 1.07 & 1.23 \\
\hline \multirow{2}{*}{ mean } & $b$ & 1.05 & 1.09 & 1.15 & 1.11 & 1.02 & 1.16 \\
& $c$ & 0.98 & 0.98 & 0.98 & 1. & 1.01 & 1.02 \\
\hline
\end{tabular}

substations and in particular the mean values of the power transits are kept very close to the nominal ones.

In this simulation the proposed controller is able to reduce the congestions in all the substations meaning also that the simple auto tuning procedure here presented is well performing.

\section{B. Implementation considerations}

As mentioned in the introduction, the main advantage of indirect control is the relative simplicity of its architecture, with a single price signal for all the units.

In the approach presented in this paper, the price signals delivered to the demand side units are more than one and they are function of DSRs position in the network. Two solutions for this problem are proposed here: 1) the substation is responsible to produce the overprice to apply and to send the price (or to make it available) to the units; 2 ) the substation could transmit a unique fingerprint (an ID, for example) to the units through PLC (Power Line Communication): once the unit knows the substation it belongs to, it can retrieve the power price from a central server. In this case the price signal could be computed centrally using measurements of the power transit from the operator SCADA system.

Both solutions require to add automation at substation level.

The target of the proposed control approach is to solve the technical issue of not incurring in a congestion when a low price is sent to the flexible demand. Anyway from the power system perspective, congestions have to be taken into account in phase of definition of the price or control signal otherwise the power system might not get the expected amount of down regulating power from the DSRs because the controllers are limiting the consumption.

Another aspect that has to be addressed when dealing with indirect control and congestions in the distribution network, it is that users that lie in a segment of power system which is often overloaded might not have access to very convenient energy price. A solution for this problem could consinsts in penalizing the DSO each time the substations raise the price as an incentive in order to increase the their nominal capacity.

\section{CONCLUSION}

This paper shows how controlling by prices a population of Demand Side Resources without any precautions leads to congestion in a nearly saturated distribution network.

As a solution to this problem, a local controller that produces an offset price for the loads of the substation is proposed. The local price controller has general formulation because it self tunes its parameters using historical measurements of the power transit and price signal.
Simulations are performed using building with electric radiators for space heating as demand side resources. For evaluating the impact on the grid, DSRs have been placed at the distribution level of the CIGRE' MV reference network. Three simulation scenarios have been tested: the first where the flexible demand is not used, the second where the flexible demand has been controlled using a price signal and the third where the congestion controller is operating in all the substations. The energy price of the regulating power from Nord Pool Spot market has been used as price control signal.

Simulations show that in a nearly saturated grid, indirect control of flexible demand leads to congestions. The proposed controller, when operating, is able to reduce the congestions both in terms of peak and average values showing also that the auto tuning capability performs as it should. Implementation considerations in comparison with traditional indirect control are outlined.

Also the issue of the accessibility to low energy prices for the users whom lie in nearly saturated segment of the network has been shortly addressed.

\section{REFERENCES}

[1] D. J. Hammerstrom, P. Investigator, R. Ambrosio, T. A. Carlon, J. G. Desteese, R. Kajfasz, and R. G. Pratt, "Pacific Northwest GridWise Testbed Demonstration Projects Part I . Olympic Peninsula Project," Contract, p. 157, 2007.

[2] "Flexpower project, http://ea-energianalyse.dk/projectsenglish/1027_flexpower_market_design.html."

[3] P. Nyeng and J. Ostergaard, "Information and communications systems for control-by-price of distributed energy resources and flexible demand," Smart Grid, IEEE Transactions on, vol. 2, pp. 334 -341, june 2011.

[4] F. Sossan and H. Bindner, "Evaluation of the performance of indirect control of many dsrs using hardware-in-the-loop simulations," in $C D C$, 2012 Maui, HI, US, December 2012.

[5] O. Corradi, H. Ochsenfeld, H. Madsen, and P. Pinson, "Controlling electricity consumption by forecasting its response to varying prices," Power Systems, IEEE Transactions on, vol. 28, pp. 421 -429, feb. 2013.

[6] Z. Csetvei, J. Ostergaard, and P. Nyeng, "Controlling price-responsive heat pumps for overload elimination in distribution systems," in Innovative Smart Grid Technologies (ISGT Europe), 2011 2nd IEEE PES International Conference and Exhibition on, pp. 1 -8, dec. 2011.

[7] H. M. P. Bacher, A. Thavlov, "Models for energy performance analysis," tech. rep., DTU - IMM, 2010.

[8] Y. Zong, D. Kullmann, A. Thavlov, O. Gehrke, and H. Bindner, "Active load management in an intelligent building using model predictive control strategy," in PowerTech, 2011 IEEE Trondheim, pp. 1 -6, june 2011.

[9] C. T. F. C6.04.02, "Benchmark systems for network integration of renewable and distributed energy resources," tech. rep., Cigre' Internationl Council on large electric systems, July 2009. 